\title{
Perceived Control and Aging: A Mini-Review and Directions for Future Research
}

\author{
Stephanie A. Robinson Margie E. Lachman \\ Department of Psychology, MS 062, Brandeis University, Waltham, MA, USA
}

\section{Keywords}

Perceived control · Control beliefs - Lifespan development . Aging $\cdot$ Health $\cdot$ Cognition

\begin{abstract}
This brief review on perceived control and aging is organized according to 3 perspectives of research involving description, explanation, and modification. An extensive body of literature has utilized cross-sectional and correlational methods to describe the sociodemographic variations and outcomes associated with perceived control. This work has focused on differences in perceived control as a function of age, sex, education, socioeconomic status, and culture and has identified positive associations with many aging-related outcomes involving health and well-being. With growing evidence regarding the health benefits of perceived control in the context of a declining sense of control with aging, there has been an increased effort to uncover the mechanisms involved, with the hopes of developing methods to maintain and/or promote adaptive control beliefs throughout adulthood. Through longitudinal and experimental work, researchers are beginning to clarify the directionality and elucidate the mechanisms to explain the associations. Recent evidence from longitudinal studies shows that control beliefs have an impact on subsequent changes in health. Yet, the findings suggest that it is not a unidirectional relationship. A conceptual model suggesting an ongoing recip-
\end{abstract}

\section{KARGER}

(C) 2017 S. Karger AG, Basel

E-Mail karger@karger.com

www.karger.com/ger rocal relationship between perceived control and health and well-being is discussed. Research examining the mechanisms that link perceived control to aging-related outcomes can help to inform and to develop effective interventions that are tailored to the individual's specific barriers and goals. We consider new directions for research, including more attention to intraindividual variability and reactivity to daily challenges, such as stress, with the goal of advancing our understanding of how perceived control contributes to aging-related outcomes. More work is needed to develop strategies to enhance control beliefs in later life. Although it will not always be possible to modify control beliefs, researchers can take these beliefs into account when developing interventions. A personalized approach is recommended as a way to tailor interventions that are compatible with individuals' beliefs about control to facilitate adaptive behavior change. Conclusions focus on selected issues and considerations for future research.

(c) 2017 S. Karger AG, Basel

\section{Introduction}

Over the past 50 years, since Julian Rotter's [1] first writings on the locus of control, a wealth of research has helped to describe and explain the relationship of control beliefs to aging and various health (psychological, physical, and cognitive) outcomes. This brief review will sum-

Stephanie A. Robinson

Department of Psychology, MS 062, Brandeis University PO Box 549110

Waltham, MA 02454 (USA)

E-Mail Srobins1@brandeis.edu 
marize some of the key themes from this large body of research and will explore future avenues with a focus on interventions to modify control beliefs. We organize our review of perceived control and aging in terms of 3 research goals: description, explanation, and modification. Descriptive work has largely focused on how perceived control varies as a function of demographic factors, such as age, sex, socioeconomic status (SES), and culture. In addition, this work has examined correlations of control beliefs and key aging-relevant domains, such as cognitive functioning and physical health. The explanatory work has looked to understand mechanisms that link control beliefs with health and cognitive outcomes with longitudinal and experimental designs. In addition, this work has considered the antecedents of changes in control beliefs. Finally, the work on modification has focused on developing interventions to change control beliefs. This work can not only help to identify ways to optimize perceived control but can also uncover some of the mechanisms that contribute to changes in control beliefs. Suggestions for future directions will be presented, with a focus on intraindividual variability and new frontiers using personalized approaches to optimize health and well-being.

\section{Descriptive Studies}

Perceived control, or one's beliefs about the likelihood that one's actions can bring about desired outcomes, is studied in various forms with varying nomenclature (e.g., self-efficacy, sense of control, personal mastery, control beliefs, locus of control, learned helplessness, primary and secondary control, etc.) [2]. While there are subtle differences, these terms all assess one's expectations about the extent to which one can bring about desired outcomes. This includes beliefs about one's abilities or efforts as well as expectancies about the constraints or obstacles that may interfere with or prevent successful goal attainment. Control beliefs are operationalized with self-assessments using items or statements that focus on rating the degree to which one expects to be able to bring about desired outcomes or to overcome external constraints in order to reach goals, in general or within specific domains and situations [3]. Although Rotter [1] initially conceptualized locus of control as a stable, generalized, and unidimensional construct, much has been written about variations in beliefs across domains and the multidimensionality of control beliefs (e.g., personal mastery, perceived constraints) $[2,4]$. Additionally, while control beliefs are largely studied in terms of individual differences between persons, there is evidence for within-person changes over both the short and long term [e.g., 4, 5]. Despite the array of conceptual and measurement differences in the perceived control construct, there are consistent sociodemographic variations and relationships with health and cognitive outcomes. The control variable has been studied both as an independent and dependent variable, as well as a moderator.

\section{Perceived Control and Age}

Much of the early work on perceived control was correlational or cross-sectional and primarily focused on children and college students. The late 1970s marked the start of investigations of perceived control within the context of aging; however, this early work was primarily focused on older adults in nursing homes [6]. The construct of perceived control was subsequently considered by a growing number of gerontologists and lifespan developmental psychologists, leading to a more comprehensive understanding of the role of control beliefs and expectancies for the aging process [4,7-9].

What was once primarily treated as a stable individual difference variable was explored as a function of aging. Recent longitudinal work has provided the opportunity to gain a firmer understanding of the development of control across the lifespan. Although one's level of perceived control may vary across domains and as a function of sociodemographic characteristics (discussed below), control beliefs, in general, show a curvilinear pattern over the life course, with a peak in midlife and declines at an accelerated rate in later adulthood [8-11]. Such declines in perceived control are related to the increasing pervasiveness of age-related obstacles and limitations, such as biological and social changes in available resources (e.g., health, social status, income, and bereavement) [3]. This decrease in later life can lead to heightened vulnerability with regard to health and longevity $[4,12]$.

A realistic appraisal of one's situation with regard to controllability is a hallmark of successful aging. The motivational theory of lifespan development, proposed by Heckhausen et al. [7], describes how adults adapt to agerelated challenges across the lifespan and maintain their psychological and physical health. It theorizes that specific control strategies associated with goal engagement and disengagement processes represent central motivational responses that can promote adaptation to age-related challenges. Such control strategies are adaptive when used congruently with opportunities for the attainment of personal goals or overcoming a problem [13]. Furthermore, this theory states that outcomes can be op-
436

Gerontology 2017;63:435-442

DOI: $10.1159 / 000468540$
Robinson/Lachman 
timized if individuals adjust their behavioral and cognitive control strategies to age-related changes in control capacity (e.g., by devoting effort to feasible goals or by reducing effort to unattainable goals) [14]. An interesting question for future research is whether and how control beliefs are related to the usage of control strategies [15].

\section{Sociodemographic Variations}

Control beliefs also vary as a function of sex, SES, and culture. In general, women tend to report lower perceived control than men. However, these differences in sex appear to be less pronounced among those with a college education, and there is some evidence that women report a higher sense of control than men in some domains (e.g., social, marriage) $[4,16]$. The relationship between perceived control and SES is well established, wherein those with greater education or higher income, on average, perceive greater control [8]. Lower SES is associated with lower levels of perceived control, likely due to greater environmental constraints associated with lower income or less education, yet there are individual differences within SES groups $[4,16,17]$. One consistent and important finding is that sense of control moderates the relationship of SES and health [16]. Survey studies using representative samples have demonstrated that greater mastery and lower perceived constraints were related to better health, greater life satisfaction, and lower depressive symptoms [16]. While those with a lower SES typically show lower levels of perceived control, those with a lower SES who do show higher levels of perceived control tend to have health and longevity comparable to those who have a higher SES $[16,18]$. Thus, a sense of control mitigates the SES differences in health and mortality risk.

With regard to directionality, a recent study found evidence that participants who were 18 years of age with a higher sense of control were more likely to pursue a university education than those with lower control beliefs [19]. Thus, control beliefs may drive educational attainment. However, in this same study, the educational level of one's parents also was found to predict the control beliefs of their offspring, suggesting that education has an impact on control beliefs across generations.

Not only do levels of perceived control differ by culture, the apparent benefits differ by culture as well. According to the Pew Research Center, those from the United States had the greatest percentage reporting that they are in control of life outcomes across 44 countries [12]. Interestingly, cross-cultural work has shown that, while the Japanese and Americans have similar levels of control beliefs, they are more important for the well-being of

Perceived Control and Aging
Americans than for the Japanese [20]. It is believed that cultural differences in perceived control are likely related to variations in economic conditions, values, and religious beliefs [4].

\section{Health, Well-Being, and Cognitive Correlates}

There are many well-established associations of perceived control with health and well-being. "It is consistently found that a high sense of control is associated with being happy, healthy, wealthy, and wise" [p. 283, 9]. Perceiving more control serves as a protective factor for psychological and emotional well-being [21, 22], as well as better health, including longevity $[18,23]$. Furthermore, it is associated with enhanced cognitive outcomes, such as mitigated memory decline [24], more adaptive strategy use [25], and greater effectiveness of cognitive training $[26,27]$.

\section{Explanatory Studies}

\section{Longitudinal Studies}

Perceived control plays an important role for health and well-being across the lifespan; it is linked to performance in multiple domains and can buffer some of the deleterious effects of aging. Considering these benefits that have been found in descriptive work, another research approach has been to examine explanatory factors and mechanisms involved in linking perceived control to health and well-being. The directionality of the relationship between control beliefs and health outcomes (physical, cognitive, and psychological) has been of heightened interest: does perceived control lead to enhanced health; do those with better health outcomes perceive more control; if one experiences health problems, does that lead to a decline in one's sense of control? Longitudinal studies can help to disentangle this relationship [18,23]. There is growing longitudinal evidence that suggests that perceived control influences changes in health. For example, Infurna et al. [28], using data from the Americans' Changing Lives study, examined the relationship between perceived control and health over 15.5 years in adults 25-96 years of age. They found that perceived control predicted subsequent changes in self-reported health conditions in older adults (but not in middle-aged adults). They did not find evidence for the reverse direction (i.e., health predicting changes in control), suggesting that control beliefs lead to better health outcomes [28].

However, there is support for a cyclic relationship in that perceiving control can serve both as a predictor and

Gerontology 2017;63:435-442

DOI: $10.1159 / 000468540$ 
an outcome of health and cognition [10,29]. For instance, older adults who experience declines in memory or are met with physical limitations may consequentially experience a lowered sense of control in these domains, particularly if these changes can be ascribed to uncontrollable factors. Subsequently, this lowered sense of control can result in worse cognitive or physical functioning [4]. As an example, Infurna et al. [23] examined the extent to which level of and change in perceived control over 16 years predicted health. They found evidence for bidirectional associations, where more perceived control predicted higher well-being and better health, and better well-being and health predicted more perceived control 16 years later [23].

\section{Mechanisms}

Soederberg Miller and Lachman [30] proposed a conceptual model, updated by Lachman [12] in 2006, describing some of the possible mechanisms involved in the relationship between perceived control and performance. Mechanisms that underlie the relationship between perceived control and many of the positive health outcomes outlined above include emotion regulation, engagement in health-promoting behaviors, social support, and stress reactivity [12, 31, 32]. In particular, feeling in control can help people regulate their emotions and adapt to significant life events and setbacks $[33,34]$ and to processes that threaten health outcomes, such as disease or mortality [35]. Additionally, individuals who view their health as more controllable are more likely to adopt and maintain health-promoting behaviors, such as exercising regularly or going to preventative doctor's appointments $[36,37]$. In terms of social support, perceived control may help people to be more proactive about seeking and receiving social support, particularly during hardship, which can help to buffer the deleterious effects of stress [38]. Finally, perceived control may indirectly influence health outcomes through reduced stress reactivity even in the face of life challenges [39]. This conceptual model, based on cognitive-behavioral theory [31], presumes these associations to be reciprocal and cyclic such that the outcomes (e.g., psychological health, physical declines, memory) influence perceived control, which subsequently can influence possible behavioral or physiological mediators and future outcomes [30, 31]. Recent empirical research has begun to directly test these mediational models linking control and relevant outcomes. For example, Infurna and Gerstorf [24] found that physical and behavioral factors, such as physical activity, physical fitness, and cardiovascular health, played a mediating role in the relationship between perceived control and memory.

\section{Modifying Control: Experimental and Intervention Studies}

Experimental work on control beliefs is crucial not only to establishing causality by examining explanatory factors but also to help develop interventions to promote control beliefs. It is first necessary to establish whether perceived control can be modified. Although often considered a stable trait, some studies have demonstrated that experimental manipulations can result in change to one's perceived level of control, suggesting its plasticity or malleability [4].

\section{Interventions to Promote Perceived Control}

The abundance of evidence for the relationship between greater perceived control and enhanced health and well-being, along with aging-related declines in control, highlights a need for control-promoting interventions. Intervention work can help to better understand the mechanisms underlying the outcomes and benefits of perceived control. There is a current trend to establish interventions that can enhance one's level of perceived control, with the hope that this will result in positive health outcomes. For example, Zautra et al. [40] were able to minimize depressive symptoms, negative affect, and catastrophizing using an intervention aimed at enhancing one's perception of personal control based on cognitive behavioral therapies. Some of these intervention efforts to promote perceived control have focused on cognitive restructuring, a method drawn from cognitive therapy that helps to identify, challenge, and alter maladaptive beliefs, as a way to reframe misconceptions about controllability over desired outcomes [4]. Other interventions have focused on increasing one's choice, flexibility, and support in one's environment as a means towards increasing control [3]. As an example, Langer and Rodin's [6] classic study provided nursing home residents with more control over their environment by allowing them to take care of their own plant and schedule what time they wanted to watch movies. Providing choices resulted in enhanced well-being, activity, and health, although the researchers did not directly assess whether there were changes in perceived control.

It is also possible to enhance perceived control in specific domains [e.g., 23, 41]. For example, many adults feel a lower sense of control over their aging process. That is, they may presume that it is too late for them to learn or try new things or that they cannot improve their performance or functioning in specific domains associated with aging-related declines (e.g., memory or physical
438

Gerontology 2017;63:435-442

DOI: $10.1159 / 000468540$
Robinson/Lachman 
ability). Older adults with low control beliefs often see physical decline as unavoidable and irreversible with advancing age, i.e., uncontrollable. As such, any motivation for behavior change may be low because it is expected that interventions to promote physical decline, such as exercise, will not do any good or may even cause harm. Additionally, many adults may not have the requisite skills to change their routines. These 2 factors (one's perceived control over one's ability to change and one's actual skill set) are crucial to consider when designing interventions.

Past work on behavior change in older adults suggests that maladaptive beliefs about aging may impede success if the intervention is only focused on improving skill performance (e.g., adding an exercise routine) [31]. Intervention studies observing the effect of self-efficacy (or one's beliefs in one's ability to perform a given behavior, an important component of perceived control) on responsiveness to training provide further evidence of the relationship between self-efficacy beliefs and performance. In the context of physical activity, researchers have found that higher levels of self-efficacy were predictive of increases in exercise behavior and maintenance of the exercise regimen [42]. In contrast, in the ACTIVE cognitive training trial, higher initial levels of cognitive performance were related to less decline in cognitive-specific self-efficacy, not the other way around [43]. Nevertheless, when examining cognitive interventions, a sense of control may play a role in their effectiveness [44, 45]. Therefore, comprehensive interventions that target both skills training and expectancies, such as self-efficacy and perceived control, may be the most efficacious [12, 43]. This multifaceted approach has been demonstrated in past work [46]. For example, Lachman et al. [41] examined how cognitive restructuring and memory skills training, both combined and individually, influenced perceived control and memory performance in older adults. They found that cognitive restructuring about memory controllability was most effective for increasing perceived control over memory when it was combined with memory skills training, suggesting that older adults appear to need some direct intervention focused on beliefs, such as developing awareness of the potential for improvement $[41,43]$. Another example of the success of a multimodal intervention can be seen in A Matter of Balance program, which used similar cognitive restructuring techniques to reframe control beliefs and was successful in changing control beliefs and intended activity and in reducing fear of falling [46].

Perceived Control and Aging

\section{Future Directions}

Despite the vast amount of work that has explored the links of perceived control to positive health outcomes, there is still work to be done to fully understand the processes involved and to work towards optimizing control. There are individual differences in perceptions of control even given the same objective circumstances. Understanding what leads some but not others to perceive a situation as controllable or not has strong repercussions for one's physiological, behavioral, and emotional health [31]. There are also intraindividual variations in perceived control, that is, changes within persons under different circumstances. Future research from an interdisciplinary perspective is needed to understand the biopsychosocial processes involved, as this may lead to promising insights and interventions.

\section{Intraindividual Variability}

Once thought of as a stable trait that merely varied between persons due to individual differences, evidence suggests that perceived control, in fact, also varies intraindividually (i.e., within persons). There have been great strides to explore how perceived control changes across the lifespan, and recently, there has also been interest in exploring these variations on a more shortterm basis [3]. In fact, regardless of the average level of perceived control, the stability of one's level of perceived control predicts longevity and cognitive performance $[47,48]$.

The ability to maintain a relatively high sense of control even in the face of failure or loss can act as a protective factor and buffer the negative effects of stressors. Both major life stressors, such as the loss of a spouse, and minor daily stressors, such as an unexpected work deadline, have the potential to influence well-being and health [49, 50]. In particular, using a daily diary design, Neupert et al. [51] explored how differences in control beliefs influenced physical and emotional reactivity to daily stressors across different domains (interpersonal, work, network, and home). Results indicated that perceiving fewer constraints was related to less emotional and physical reactivity to daily interpersonal stressors and that having a greater sense of mastery was related to less physical reactivity to work stressors and less emotional reactivity to network stressors. Infurna and Reich [52] suggest that exploring perceived control in the context of stressors is an important avenue, as perceived control can play a crucial role in helping one to overcome stress. Future work should continue to explore strategies to maintain or increase one's

Gerontology 2017;63:435-442 DOI: $10.1159 / 000468540$ 
sense of control and perhaps to reduce variability even when confronted with daily challenges.

\section{Personalized Interventions}

While personalized medicine has typically focused on targeted medical treatments, a similar individual-based approach for developing effective interventions to optimize control beliefs could help promote adaptive health and positive aging-related outcomes. Though past work has investigated interventions to promote perceived control, it is important to take individual differences into account in order to personalize interventions. Personalized interventions have been successful in reducing risky health behaviors (e.g., alcohol consumption) and promoting healthy behaviors (e.g., smoking cessation, selfmanagement of type II diabetes) [53-55]. By considering individual differences in control beliefs, interventions can be established that are relevant to the unique goals or barriers of the individual. This personalized approach considers that there are types or clusters of people with commonalities who would respond to particular treatment approaches in similar ways. Thus, the goal is to tailor the treatments accordingly with the goal of sustained behavior change.

We propose that a personalized approach could be helpful in creating effective interventions for behavior change, such as increasing physical activity. For example, if an older adult's physician suggests an increase in physical activity, it is important to identify the specific barriers that might prevent him or her from engaging in physical activity. If a low sense of control about physical activity (e.g., "I'm too old to exercise" or "I can't find the time to exercise") is suggested, then the intervention could start by targeting the sense of control. This could include messages about the benefits of physical activity at any age or ways to increase activity levels with small changes to one's routines, along with providing the necessary skills and environmental supports to engage in more physical activity. On the other hand, if the main barrier to becoming more active is lack of knowledge about how to exercise, rather than a low sense of control, this would suggest a focus on strategies to obtain the necessary information and skills, as a means to boosting exercise self-efficacy.

Ultimately, these changes in beliefs and behavior are expected to have long-term benefits for performance (e.g., cognitive), psychological well-being (e.g., affect), and health outcomes (e.g., fitness). Although it is not always possible to modify control beliefs, researchers could take such beliefs into account when developing interventions. For those who are resistant to changing beliefs, the focus may be adapted to target environmental manipulations that create a sense of control without necessarily directly changing beliefs. Such environmental manipulations are exemplified in Langer and Rodin's [6] notable study, where giving someone control over their environment (e.g., the ability to make choices about their living situation) can foster a sense of well-being. Finally, it is important to acknowledge that those who enroll in interventions are likely those who already have a relatively strong sense of control. Targeting those who are low in perceived control may require a form of intervention to get them to participate in the first place. Differences in beliefs about control can have a critical impact on the effectiveness of interventions, such as one's willingness to enroll in the intervention and one's receptivity to and success with the intervention.

\section{Other Considerations}

Objective versus Subjective Control

While this mini-review has concentrated on subjective assessments, i.e., perceived control, it is also important to consider the role of objective control $[3,56]$. Previous work has indicated that conflict between environmental affordances of control and personal beliefs of control can lead to negative psychological, physiological, and behavioral outcomes which, in turn, correspond to poor performance, passivity, and depression [57]. As outlined in Baltes's model of learned dependency [58], a lack of systematic and predictable connections between behavioral and environmental events leads to learning that these behavioral events do not result in distinct outcomes (i.e., noncontingency). In line with Weiner's [59] attributional theory, an individual can perceive this lack of predictable connections between behavior and consequences as failure and may attribute it to their own incompetence, an unresponsive environment, or both. To those with such attributions, noncontingency is believed to lead to a perceived lack of control and, in turn, to learned dependency [58]. Baltes [58] has demonstrated that this type oflearned dependency can indeed be modified. Specifically, in a series of studies, she implemented a training program geared toward providing social partners of the elderly with skills that would increase the exhibition of independence-supportive behaviors, resulting in an environment that continues to be responsive to dependency when appropriate but stimulates and fosters independence.

Other work on the interplay of objective and perceived control, conducted by Agrigoroaei et al. [56], manipulated objective control with a driving simulation program and explored how objective and subjective control inter-
440

Gerontology 2017;63:435-442

DOI: $10.1159 / 000468540$
Robinson/Lachman 
act to influence one's response to stress. They assessed general control beliefs before exposing participants to either a normal driving simulation (normal controllability) or an unpredictable simulation (low controllability) where control over steering and braking was reduced. They demonstrated that the effects on stress from manipulating objective control depended on the participants' level of perceived control. Specifically, compared to participants who had a lower perception of control, participants who had a higher perception of control had a larger stress response in the low controllability condition. This increased stress response may be representative of compensatory vigilance to counteract the low level of objective control [56]. This work further confirms the importance of investigating how one's perception of control impacts one's health and daily life in the context of environmental contingencies and control [3].

\section{The Downside of Perceived Control}

While the benefits of perceived control are well established, it is important to note that having a high sense of control can also be deleterious in some circumstances, such as when objective control is low. For example, those who perceive greater control over their health may be more likely to ignore actual health problems or not regularly go to the doctor [3]. This paradox is especially true when one perceives one's health as controllable but devalues the importance of it. This combination of evaluations may cultivate an ill-advised and erroneous sense of invincibility [60]. In fact, it has been suggested that a realistic assessment of one's control may be more beneficial than optimistic overestimations. That is, knowing when to relinquish control may be a form of wisdom and may be associated with increased well-being [4].

\section{Conclusions}

Perceived control is positively associated with a multitude of important outcomes related to health and wellbeing. A depth of research has diligently described these outcomes, such as enhanced cognitive functioning, physical health, and even longevity. Using longitudinal and experimental designs, researchers have begun to explain the underlying mechanisms involved in these relationships, suggesting a dynamic relationship in which greater control beliefs lead to greater engagement in adaptive strategies and health-promoting behaviors resulting in better health, which in turn can lead to increases in perceived control. Future work should continue to explore the best strategies to modify control beliefs. By investigating the mechanisms as well as what contributes to shortterm variability in control beliefs, researchers can develop a deeper understanding which can help to inform effective interventions that are tailored to the individual's specific barriers and goals.

\section{Acknowledgements}

This review was supported in part by NIA grants RO1 AG 17920 and P30 AG048785 and NRSA Institutional Training Grant 5T32AG000204.

\section{References}

1 Rotter J: Generalized expectancies for internal versus external control of reinforcement. Psychol Monogr Gen Appl 1966;80:1-28.

2 Skinner EA: A guide to constructs of control. J Pers Soc Psychol 1996;71:549-570.

3 Lachman ME, Agrigoroaei S, Rickenbach EH: Making sense of control: change and consequences. Emerg Trends Soc Behav Sci 2015, DOI: 10.1002/9781118900772.etrds0209.

4 Lachman M, Neupert SD, Agrigoroaei S: The relevance of control beliefs for health and aging; in Schaie KW, Willis SL (eds): Handbook of the Psychology of Aging. San Diego, CA, Elsevier, 2011, pp 175-190.

5 Neupert SD, Allaire JC: I think I can, I think I can: examining the within-person coupling of control beliefs and cognition in older adults. Psychol Aging 2012;27:742-749.

Perceived Control and Aging
6 Langer EJ, Rodin J: The effects of choice and enhanced personal responsibility for the aged: a field experiment in an institutional setting. J Pers Soc Psychol 1976;34:191-198.

7 Heckhausen J, Wrosch C, Schulz R: A motivational theory of life-span development. Psychol Rev 2010;117:1-53.

8 Mirowsky J, Ross CE: Life course trajectories of perceived control and their relationship to education. Am J Sociol 2007;112:1339-1382.

9 Lachman ME, Rosnick CB, Röcke C: The rise and fall of control beliefs and life satisfaction in adulthood: trajectories of stability and change over ten years. Aging Cogn Res Methodol Empir Adv 2009:143-160.

10 Infurna F, Okun M: Antecedents and outcomes of level and rates of change in perceived control: the moderating role of age. Dev Psychol 2015;51:1420-1437.
11 Drewelies J, Wagner J, Tesch-Römer C, et al: Perceived control across the second half of life: the role of physical health and social integration. Psychol Aging 2017;32:76-92.

12 Lachman M: Perceived control over agingrelated declines: adaptive beliefs and behaviors. Curr Dir Psychol Sci 2006;15:282-286.

13 Barlow M, Wrosch C, Heckhausen J, et al: Control strategies for managing physical health problems in old age: evidence for the motivational theory of life-span development; in Reich JW, Infurna FJ (eds): Perceived Control: Theory, Research, and Practice in the First 50 Years. New York, NY, Oxford University Press, 2016, pp 281-307.

14 Heckhausen J, Schulz R: A life-span theory of control. Psychol Rev 1995;102:284-304. 
15 Wrosch C, Heckhausen J, Lachman ME: Goal management across adulthood and old age: the adaptive value of primary and secondary control; in Mroczek DK, Little TD (eds): Handbook of Personality Development. Mahwah, NJ, Lawrence Erlbaum Associates Publishers, 2006, pp 399-421.

16 Lachman ME, Weaver SL: The sense of control as a moderator of social class differences in health and well-being. J Pers Soc Psychol 1998;74:763-773.

17 Wolinsky FD, Stump TE: Age and the sense of control among older adults. J Gerontol B Psychol Sci Soc Sci 1996;51:S217-S220.

18 Turiano NA, Chapman BP, Agrigoroaei S, et al: Perceived control reduces mortality risk at low, not high, education levels. Health Psychol 2014;33:883-890.

19 Vargas Lascano DI, Galambos NL, Krahn HJ, et al: Growth in perceived control across 25 years from the late teens to midlife: the role of personal and parents' education. Dev Psychol 2015;51:124-135.

20 Kitayama S, Karasawa M, Curhan KB, et al: Independence and interdependence predict health and wellbeing: divergent patterns in the United States and Japan. Front Psychol 2010;1:1-10.

21 Kunzmann U, Little T, Smith J: Perceiving control: a double-edged sword in old age. J Gerontol B Psychol Sci Soc Sci 2002;57:484-491.

22 Lachman M, Röcke C, Rosnick C, et al: Realism and illusion in Americans' temporal views of their life satisfaction: age differences in reconstructing the past and anticipating the future. Psychol Sci 2008;19:889-197.

23 Infurna FJ, Ram N, Gerstorf D: Level and change in perceived control predict 19-year mortality: findings from the Americans' Changing Lives Study. Dev Psychol 2013;49: 1833-1847.

24 Infurna FJ, Gerstorf D: Linking perceived control, physical activity, and biological health to memory change. Psychol Aging 2013;28:1147-1163.

25 Lachman ME, Andreoletti C: Strategy use mediates the relationship between control beliefs and memory performance for middle-aged and older adults. J Gerontol B Psychol Sci Soc Sci 2006;61:88-94.

26 Rebok G, Rasmusson DX, Brandt J: Prospects for computerized memory training in normal elderly: effects of practice on explicit and implicit memory tasks. Appl Cogn Psychol 1996; 10:211-223.

27 Wolinsky FD, Vander Weg MW, Martin R, et al: Does cognitive training improve internal locus of control among older adults? J Gerontol B Psychol Sci Soc Sci 2010;65:591-598.

28 Infurna FJ, Gerstorf D, Zarit SH: Examining dynamic links between perceived control and health: longitudinal evidence for differential effects in midlife and old age. Dev Psychol 2011;47:9-18.

29 Gerstorf D, Röcke C, Lachman ME: Antecedent-consequent relations of perceived control to health and social support : longitudinal ev- idence for between-domain associations across adulthood. J Gerontol B Psychol Sci Soc Sci 2011;66:61-71.

30 Soederberg Miller L, Lachman ME: The sense of control and cognitive aging. Soc Cogn Aging 1999:17-41.

31 Bandura A: Self-Efficacy: The Exercise of Control. New York, NY, WH Freeman \& Co, 1997.

32 Uchino BN: Social support and health: a review of physiological processes potentially underlying links to disease outcomes. J Behav Med 2006;29:377-387.

33 Lang FR, Heckhausen J: Perceived control over development and subjective well-being: differential benefits across adulthood. J Pers Soc Psychol 2001;81:509-523.

34 Windsor TD, Anstey KJ: Age differences in psychosocial predictors of positive and negative affect: a longitudinal investigation of young, midlife, and older adults. Psychol Aging 2010;25:641-652.

35 Pressman SD, Cohen S: Does positive affect influence health? Psychol Bull 2005;131:925971.

36 White S, Wójcicki T, McAuley E: Social cognitive influences on physical activity behavior in middle-aged and older adults. J Gerontol B Psychol Sci Soc Sci 2012;67:18-26.

37 Lachman M, Firth K: The adaptive value of feeling in control during midlife; in Brim OG, Ryff CD, Kessler RC (eds): How Healthy Are We? A National Study of Well-Being at Midlife. Chicago, University of Chicago Press, 2004, pp 320-349.

38 Antonucci TC: Social relations: an examination of social networks, social support, and sense of control; in Birren J, Schaie KW (eds): Handbook of the Psychology of Aging. San Diego, CA, Academic Press, 2001, pp 427-453.

39 Diehl M, Hay EL: Risk and resilience factors in coping with daily stress in adulthood: the role of age, self-concept incoherence, and personal control. Dev Psychol 2010;46:11321146.

40 Zautra AJ, Davis MC, Reich JW, et al: Phonebased interventions with automated mindfulness and mastery messages improve the daily functioning for depressed middle-aged community residents. J Psychother Integr 2012; 22:206-228.

41 Lachman ME, Weaver SL, Bandura M, et al: Improving memory and control beliefs through cognitive restructuring and self-generated strategies. J Gerontol Psychol Sci 1992; 47:293-299.

42 Neupert S, Lachman M, Whitbourne S: Exercise self-efficacy and control beliefs predict exercise behavior after an exercise intervention for older adults. J Aging Phys Act 2009; 17:1-14.

43 Parisi JM, Gross AL, Marsiske M, et al: Control beliefs and cognition over a 10 -year period: findings from the ACTIVE trial. Psychol Aging 2017;32:69-75.

44 West RL, Bagwell DK, Dark-Freudeman A: Self-efficacy and memory aging: the impact of a memory intervention based on self-efficacy.
Neuropsychol Dev Cogn B Aging Neuropsychol Cogn 2008;15:302-329.

45 Payne BR, Jackson JJ, Hill PL, et al: Memory self-efficacy predicts responsiveness to inductive reasoning training in older adults. J Gerontol B Psychol Sci Soc Sci 2012;67:27-35.

46 Tennstedt S, Howland J, Lachman M, et al: A randomized, controlled trial of a group intervention to reduce fear of falling and associated activity restriction in older adults. J Gerontol Psychol Sci 1998;53:384-392.

47 Eizenman DR, Nesselroade JR, Featherman DL, et al: Intraindividual variability in perceived control in an older sample: the MacArthur successful aging studies. Psychol Aging 1997;12:489-502.

48 Agrigoroaei S, Neupert SD, Lachman ME: Maintaining a sense of control in the context of cognitive challenge: greater stability in control beliefs benefits working memory. GeroPsych (Bern) 2013;26:45-49.

49 Almeida DM: Resilience and vulnerability to daily stressors assessed via diary methods. Curr Dir Psychol Sci 2005;14:64-68.

50 Infurna FJ, Luthar SS: Resilience to major life stressors is not as common as thought. Perspect Psychol Sci 2015;11:175-194.

51 Neupert SD, Almeida DM, Charles ST: Age differences in reactivity to daily stressors: the role of personal control. J Gerontol B Psychol Sci Soc Sci 2007;62:P216-P225.

52 Infurna FJ, Reich JW: Perceived control: 50 years of innovation and another 50 to go; in Reich JW, Infurna FJ (eds): Perceived Control: Theory, Research, and Practice in the First 50 Years. Oxford, Oxford University Press, 2016, pp 1-22.

53 Bierut LJ, Johnson EO, Saccone NL: A glimpse into the future - personalized medicine for smoking cessation. Neuropharmacology 2014;76:592-599.

54 Clark M, Hampson SE, Avery L, et al: Effects of a tailored lifestyle self-management intervention in patients with type 2 diabetes. $\mathrm{Br} \mathrm{J}$ Health Psychol 2004;9:365-379.

55 Neighbors C, Lee CM, Lewis MA, et al: Internet-based personalized feedback to reduce 21st-birthday drinking: a randomized controlled trial of an event-specific prevention intervention. J Consult Clin Psychol 2009;77: 51-63.

56 Agrigoroaei S, Polito M, Lee A, et al: Cortisol response to challenge involving low controllability: the role of control beliefs and age. Biol Psychol 2013;93:138-142.

57 Evans G, Shapiro DH, Lewis MA: Specifying dysfunctional mismatches between different control dimensions. Br J Psychol 1993;84: 255-273.

58 Baltes M: Dependency in old age: gains and losses. Curr Dir Psychol Sci 1995;4:14-19.

59 Weiner B: An attributional theory of achievement motivation and emotion. Psychol Rev 1985;92:548-573.

60 Chipperfield JG, Perry RP, Pekrun R, et al: The paradoxical role of perceived control in late life health behavior. PLoS One 2016;11:1-16. 